\title{
Association Between Subclinical Cardiac Biomarkers and Clinically Manifest Cardiac Diseases With Cortical Cerebral Microinfarcts
}

Saima Hilal, PhD; Yuek Ling Chai, BSc; Susanne van Veluw, PhD; Muhammad Amin Shaik, BPsych; Mohammad Kamran Ikram, PhD; Narayanaswamy Venketasubramanian, FRCP; Arthur Mark Richards, MD; Geert Jan Biessels, PhD; Christopher Chen, FRCP

IMPORTANCE Subclinical and clinical cardiac diseases have been previously linked to magnetic resonance imaging (MRI) manifestations of cerebrovascular disease, such as lacunes and white matter hyperintensities, as well as dementia. Cortical cerebral microinfarcts (CMIs), a novel MRI marker of cerebral vascular disease, have not been studied, to date, in relation to subclinical and clinical cardiac diseases.

OBJECTIVE To examine the association of blood biomarkers of subclinical cardiac disease and clinically manifest cardiac diseases with CMIs graded on 3-T MRI in a memory clinic population.

DESIGN, SETTING, AND PARTICIPANTS This baseline cross-sectional analysis of a cohort study performed from August 12, 2010, to July 28, 2015, included 464 memory clinic participants. All participants underwent collection of blood samples, neuropsychological assessment, and 3-T MRI.

EXPOSURES N-terminal pro-brain natriuretic peptide (NT-proBNP) and high-sensitivity cardiac troponin $\mathrm{T}$ (hs-cTnT) concentrations were measured by electrochemiluminescence immunoassays. Cardiac disease was defined as a history of atrial fibrillation, ischemic heart diseases, or congestive heart failure.

MAIN OUTCOMES AND MEASURES The CMIs were graded according to a previously validated protocol.

RESULTS Of 464 participants, 124 had insufficient blood plasma samples and 97 had no CMI grading (none, incomplete, or ungradable MRI), leaving a sample size of 243 for final analysis (mean [SD] age, 72.8 [9.1] years; 116 men [42.9\%]). Seventy participants (28.8\%) had cortical CMls (median, 1; range, 0-43). Compared with participants with no CMls, those with CMls had a significantly higher prevalence of atrial fibrillation (rate ratio [RR], 1.62; $95 \% \mathrm{Cl}, 1.20-21.8$ ), ischemic heart disease (RR, 4.31; 95\% Cl, 3.38-5.49), and congestive heart failure (RR, 2.05; $95 \% \mathrm{Cl}, 1.29-3.25)$. Significantly higher levels of NT-proBNP (RR, 3.16; $95 \% \mathrm{Cl}, 2.33-4.27)$ and hs-cTnT (RR, 2.17; 95\% Cl, 1.00-4.74) were found in participants with CMls. In multivariate models adjusted for demographics and vascular risk factors, higher levels of NT-proBNP (RR, 3.19; $95 \% \mathrm{Cl}, 2.62-3.90)$ and hs-cTnT (RR, 4.86; 95\% Cl, 3.03-7.08) were associated with CMIs. These associations persisted even after excluding patients with clinically manifest cardiac disease.

CONCLUSIONS AND RELEVANCE This study found that biomarkers of subclinical cardiac disease and clinically manifest cardiac diseases were associated with CMIs on 3-T MRI in patients attending a memory clinic, suggesting that cardiac disease may contribute to the development of CMls. Hence, cardiac dysfunction should be targeted as a potentially modifiable factor to prevent CMI-related brain injury.

JAMA Neurol. 2017;74(4):403-410. doi:10.1001/jamaneurol.2016.5335

Published online February 6, 2017.
Editorial page 385

Author Affiliations: Author affiliations are listed at the end of this article.

Corresponding Author: Christopher Chen, FRCP, Department of Pharmacology, Yong Loo Lin School of Medicine, National University of Singapore, 16 Medical Dr, Level 4 . Block MD3, Singapore 117600 (phccclh@nus.edu.sg). 
C ardiovascular diseases have been linked to cognitive deficits in individuals without a history of stroke or dementia. ${ }^{1}$ Ischemic heart disease, coronary artery disease, and cardiac failure have been associated with subclinical brain injury (lacunes and white matter hyperintensities), which may cause impaired cognitive performance. ${ }^{2,3}$ However, by the time cardiac diseases become clinically apparent, significant cerebral damage may have already occurred. ${ }^{4,5}$ Hence, blood biomarkers of cardiovascular disease, such as N-terminal pro-brain natriuretic peptide (NT-proBNP) and high-sensitivity cardiac troponin $\mathrm{T}$ (hs-cTnT), have recently become of interest because of their upregulation not only in myocardial stress and injury but also in the early phases of cardiovascular disease. ${ }^{6-9}$ Interestingly, higher levels of NT-proBNP and hs-cTnT were associated with cognitive impairment and dementia. $^{10}$

In addition to the traditional cerebral markers of vascular injury, such as lacunar infarcts and white matter hyperintensities, a novel marker of cerebrovascular diseases, cortical cerebral microinfarcts (CMIs), has recently gained attention because of its major role in cognitive impairment and dementia. ${ }^{11,12}$ Until recently, CMIs have not been detectable during life; however, recent advances in magnetic resonance imaging (MRI) techniques have allowed detection of CMIs in vivo. ${ }^{13,14}$ Subsequent studies have found that cortical CMIs were associated with small-vessel (lacunes, white matter hyperintensities, and microbleeds) and large-vessel (cortical infarcts and intracranial stenosis) diseases, which may suggest heterogeneous origin.. ${ }^{13}$ Furthermore, they were also linked to a history of cardiovascular disease and cognitive dysfunction. ${ }^{15}$ However, to our knowledge, the association between clinical cardiac diseases and blood markers of cardiac dysfunction with cortical CMIs on MRI have not been explored previously. We, therefore, examined the association of blood biomarkers of subclinical cardiac diseases, NT-proBNP, and hs-cTnT and clinically manifest cardiac diseases (atrial fibrillation, ischemic heart disease, and congestive heart failure) with CMIs graded on 3-T MRI in a memory clinic population.

\section{Methods}

\section{Study Population}

Participants were recruited from memory clinics in 2 study sites in Singapore (National University Hospital and Saint Luke's Hospital). Assessments of participants were performed from August 12, 2010, to July 28, 2015. Six diagnostic categories were eligible for inclusion in this study based on the following diagnoses. First, patients with no cognitive impairment were those who had no objective cognitive impairment on formal neuropsychological tests or functional loss. Second, the diagnosis of cognitive impairment no dementia was based on clinical judgment and was diagnosed in patients who were impaired in at least one cognitive domain on a formal neuropsychological test battery but did not meet the Diagnostic and Statistical Manual of Mental Disorders, Fourth Edition (DSMIV) criteria for dementia. Participants were considered to have failed a cognitive test if they scored 1.5 SDs below the educa-

\section{Key Points}

Question Is there an association between subclinical cardiac biomarkers and overt cardiac diseases with cortical cerebral microinfarcts?

Findings In this cross-sectional analysis of 243 individuals from a cohort study of 464 participants, higher levels of $\mathrm{N}$-terminal pro-brain natriuretic peptide and high-sensitivity cardiac troponin T were associated with cortical cerebral microinfarcts. Atrial fibrillation, ischemic heart disease, and congestive heart failure were also independently linked with cortical cerebral microinfarcts.

Meaning In a memory clinic setting, cardiac dysfunction should be targeted as a potentially modifiable factor to prevent cortical cerebral microinfarct-related brain injury.

tional level-adjusted cut-off values on each test. Failure in at least half the tests in each domain was considered impairment in that domain. Third, vascular cognitive impairment no dementia was defined as a history of ischemic stroke within the past 6 to 24 months and neuroimaging evidence of cerebral infarction, with objective evidence of neuropsychological deficits. ${ }^{16}$ Fourth, dementia was diagnosed according to DSM-IV criteria. The etiologic diagnoses of dementia were based on internationally accepted criteria: Alzheimer disease was diagnosed using the National Institute of Neurological and Communicative Disorders and Stroke and the Alzheimer Disease and Related Disorders Association; Alzheimer disease with cerebrovascular disease or mixed dementia was defined as fulfilling the National Institute of Neurological and Communicative Disorders and Stroke and the Alzheimer Disease and Related Disorders Association criteria together with concomitant cerebrovascular disease on MRI (presence of multiple infarcts or extensive white matter hyperintensities) ${ }^{17}$; and vascular dementia was defined using the National Institute of Neurological Disorders and Stroke and Association Internationale pour la Recherché et l' Enseignement en Neurosciences criteria. ${ }^{18}$

All participants underwent physical, clinical, and neuropsychological assessments and neuroimaging at the National University of Singapore. ${ }^{10}$ Ethics approval for this study was obtained from National Healthcare Group Domain-Specific Review Board. The study was conducted in accordance with the Declaration of Helsinki. ${ }^{19}$ Written informed consent was obtained, in the preferred language of the participants, by bilingual study coordinators before recruitment. All the data were deidentified before study assessments.

\section{Blood Tests}

The NT-proBNP and hs-cTnT concentrations were measured by electrochemiluminescence immunoassays using the NTproBNP and hs-cTnT assays on an automated Cobas e411 analyzer (Roche Diagnostics GmbH). Quality controls included in both were within lot-specific target ranges and within the range of concentrations detected in the samples from 5 to 20627 $\mathrm{pg} / \mathrm{mL}$ (to convert to nanograms per liter, multiply by 1 ) for NTproBNP and 0.003 to $0.20 \mathrm{ng} / \mathrm{mL}$ (to convert to micrograms per liter, multiply by 1) for hs-cTnT. 


\section{Cardiac Diseases}

A history of clinically manifest cardiac disease (ie, atrial fibrillation, ischemic heart disease, and congestive heart failure) was verified from medical records in which data on chest radiography, angiography, electrocardiography, echocardiography, cardiac catheterization, and hospital course for those who were admitted were available. Atrial fibrillation was defined as abnormal heart rhythm characterized by rapid and irregular heart rate on electrocardiography. Ischemic heart disease encompassed a history of angina or myocardial infarction. Congestive heart failure was diagnosed based on the European Society of Cardiology criteria, including symptoms and/or signs consistent with heart failure together with echocardiographic evidence of abnormal systolic and/or diastolic function. ${ }^{20}$

Demographic and Other Vascular Risk Factor Assessment A detailed questionnaire was administered to all participants to collect information on age, sex, race, educational level, and smoking history. A history of hypertension, hyperlipidemia, and diabetes was also noted and subsequently verified by medical records. Physical examination included height, weight, and blood pressure. Systolic and diastolic blood pressures were measured using a digital automatic blood pressure monitor after the participant rested for 5 minutes. Blood pressure was measured twice, 5 minutes apart. The mean of 2 readings was considered as the relevant blood pressure. Hypertension was defined as systolic blood pressure of $140 \mathrm{~mm} \mathrm{Hg}$ or higher and/or diastolic blood pressure of $90 \mathrm{~mm} \mathrm{Hg}$ or higher or prescription of antihypertensive medication. Mean arterial blood pressure was calculated as two-thirds of the diastolic blood pressure plus one-third of the systolic blood pressure. Type 2 diabetes was defined as a glycated hemoglobin level of $6.5 \%$ or higher (to convert to proportion of hemoglobin, multiply by 0.01) or use of antidiabetic medication. Hyperlipidemia was defined as total cholesterol levels of $160 \mathrm{mg} / \mathrm{dL}$ (to convert to millimoles per liter, multiply by 0.0259 ) or use of lipidlowering medication.

\section{Neuroimaging}

Magnetic resonance imaging was performed on a 3-T Magnetom Trio Tim scanner (Siemens Corp), using a 32-channel head coil, at the Clinical Imaging Research Centre of the National University of Singapore. Participants with claustrophobia, those with contraindications for MRI, or those who were unable to tolerate the procedure were excluded. For each participant, the following MRI markers were determined.

\section{Cortical CMIs}

Cortical CMIs were graded on T1- and T2-weighted and fluidattenuated inversion recovery (FLAIR) sequences and were defined as hypointense lesions on T1-weighted images, less than $5 \mathrm{~mm}$ in diameter, restricted to the cortex, and perpendicular to the cortical surface. The location of a hypointense cortical lesion found on T1-weighted images was confirmed on FLAIR and T2-weighted images (Figure). The lesion was rated as a definite cortical CMI if it was hyperintense or isointense (with the surrounding tissue) on FLAIR and T2-weighted images. The lesion was discarded as a cortical CMI if at the same location a hypointense signal was found on FLAIR or T2-weighted images, indicating the T1-weighted hypointense lesion was attributable to a hemorrhagic lesion (confirmed on susceptibility weighted imaging sequence), a vessel, or an artifact. ${ }^{15}$ Dilated perivascular spaces that extended from the white matter into the cortex, producing similar cortical signal intensities to those of cortical CMIs, were not considered. ${ }^{14}$ Similarly, cortical CMIs in areas affected by large cortical infarcts were also discarded.

\section{Other MRI Markers}

Quantitative MRI analyses were performed at the Department of Medical Informatics, Erasmus University Medical Center, Rotterdam, the Netherlands. Total intracranial volume and white matter hyperintensity volume of the whole brain were quantified by automatic segmentation using the T1-weighted and FLAIR sequences. ${ }^{21}$ Cortical and lacunar infarcts were graded on FLAIR and T2-weighted sequences using the Standards for Reporting Vascular Changes on Neuroimaging criteria. ${ }^{22}$ Cerebral microbleeds were graded using the Brain Observer Microbleed Scale. ${ }^{23}$ Intracranial stenosis was graded on magnetic resonance angiography and was defined as stenosis of $50 \%$ or more in the vertebral, basilar, internal carotids, posterior cerebral, middle cerebral, and/or anterior cerebral arteries.

\section{Statistical Analysis}

Differences in the baseline characteristics of included and excluded participants were explored using the $t$ test and $x^{2}$ test. Trends in baseline characteristics across different categories of CMI numbers were examined using analysis of variance for normally distributed data and the Kruskal-Wallis test for skewed variables, and a $P$ value for trend was computed. The NT-proBNP and hs-cTnT levels were logarithmically transformed to ensure a normal distribution for regression analysis. For all models, NT-proBNP and hs-cTnT levels and cardiac diseases were treated as determinants and CMI on MRIs as outcome. To examine the robustness of the associations, CMIs were included in these models as (1) CMI counts, (2) presence vs absence of CMIs, and (3) multiple ( $\geq 3$ ) vs less than 3 CMIs. Poisson regression models were constructed for CMI counts with measure of association as rate ratios (RRs) and corresponding 95\% CIs. For the Poisson regression model, interpretation of the effect sizes was as follows: a person with a 10fold (1 unit of 10-log [cardiac marker] increment) increase in cardiac biomarker level will be RR times (eg, 4 times) more likely to have 1 additional CMI on MRI compared with a person with a lower biomarker level. For categorical outcomes, logistic regression models were computed with odds ratios (ORs) and 95\% CIs. Last, to examine whether the association between subclinical cardiac biomarkers and CMIs remained independent of clinically manifest cardiac diseases, Poisson and logistic regression models were again constructed. All the models were initially adjusted for age and sex and subsequently for vascular risk factors. Two-sided $P<.05$ was considered statistically significant. Statistical analysis was performed using standard statistical software (SPSS, version 23, SPSS Inc). 
Figure. Varying Numbers of Cortical Cerebral Microinfarcts (CMIs) on 3-T Magnetic Resonance Imaging in Different Patients

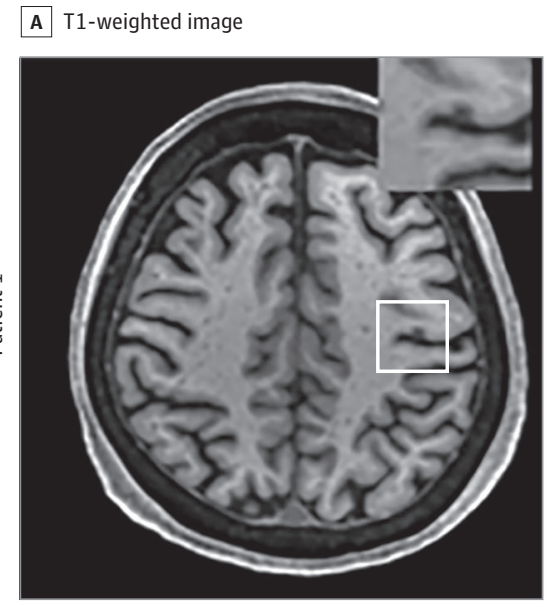

D T1-weighted image

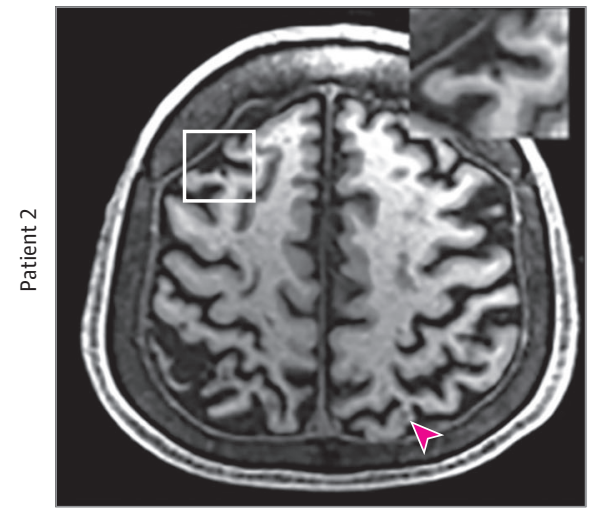

G T1-weighted image

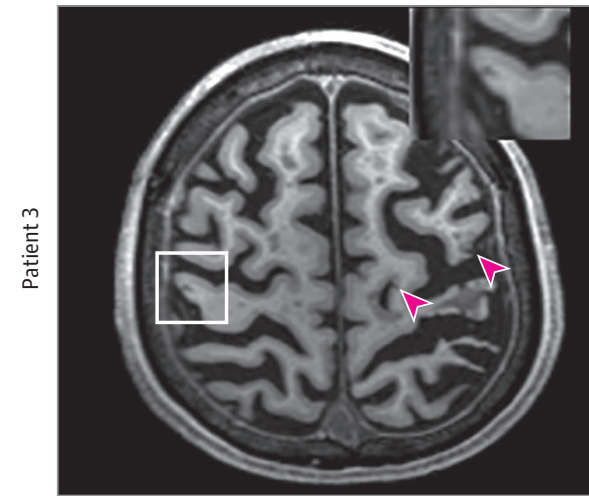

B FLAIR image

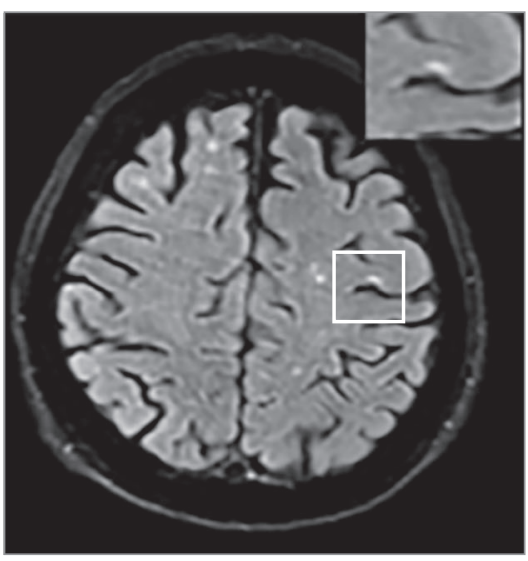

E FLAIR image

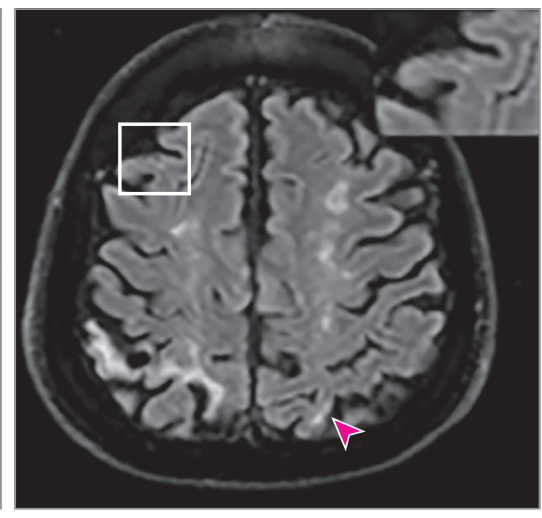

H FLAIR image

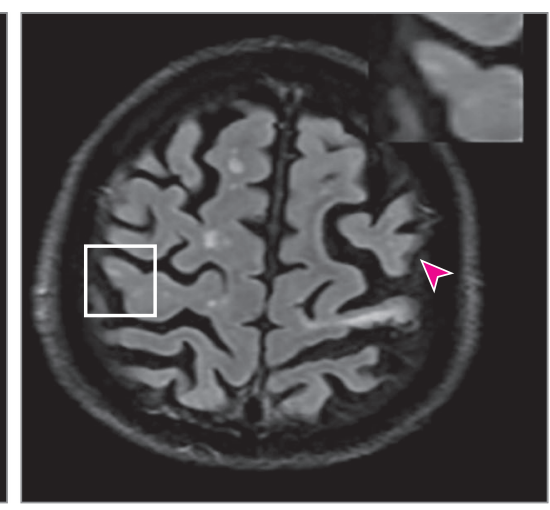

C T2-weighted image

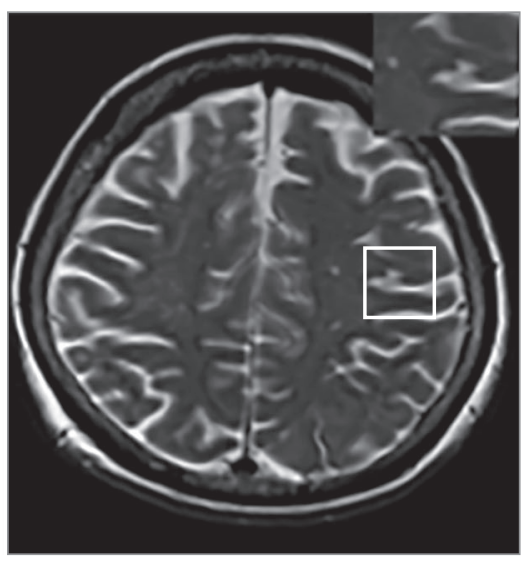

F T2-weighted image

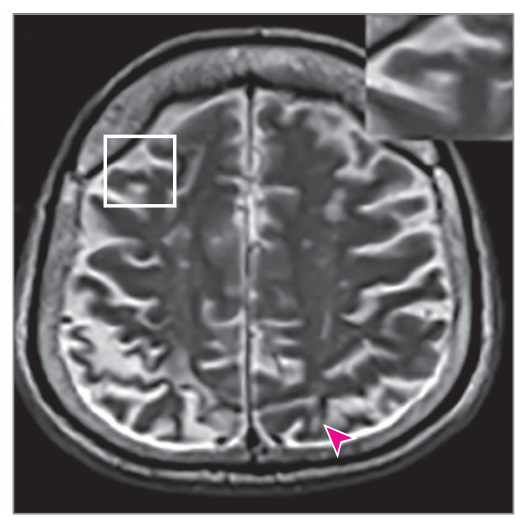

I T2-weighted image

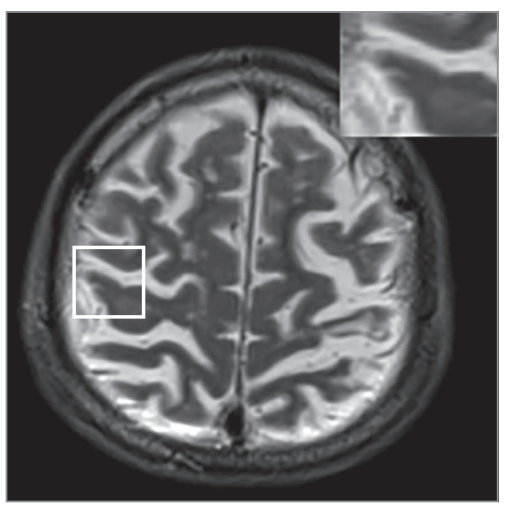

A representative cortical $\mathrm{CMI}$, which was first explored on a 3-dimensional T1-weighted image (A, D, G) and further confirmed on fluid-attenuated inversion recovery $(F L A I R)(B, E, H)$ and $T 2$-weighted $(C, F, I)$ images.

\section{Results}

Of 464 participants, 124 had insufficient blood plasma samples and 97 had no CMI grading (none, incomplete, or ungradable MRI), leaving a sample size of 243 for final
White boxes indicate area with CMls as shown in the magnified view in insets: red arrowheads, additional CMls other than the one shown in white boxes.

analysis (mean [SD] age, 72.8 [9.1] years; 116 men [42.9\%]). Those who were excluded were not significantly different from those included in the analysis (Table 1). Among 243 participants, $70(28.8 \%)$ had 1 or more cortical CMIs. Of the participants with cortical CMIs, 36 (51.4\%) had 1, 20 (28.6\%) had 2 to 4 , and 14 had 5 or more CMIs, with a range of 0 to 
43. Table 2 gives the baseline characteristics of participants across increasing numbers of CMIs. In brief, an increasing frequency of hypertension, hyperlipidemia, subclinical cardiac biomarkers, and cardiac diseases was observed in the CMI groups. Compared with participants without cortical CMIs, the prevalence of small-vessel (lacunes and cerebral microbleeds) and large-vessel (cortical infarcts and intracranial stenosis) diseases on MRI was higher in persons with cortical CMIs.

Table 3 details the association of subclinical cardiac biomarkers and clinically manifest cardiac diseases with cortical CMIs graded on MRI. Higher levels of NT-proBNP and hs-cTnT were significantly associated with an increasing number of cortical CMIs in age- and sex-adjusted models.
After additional adjustments with vascular risk factors, these associations remained significant. Similarly, a significant association was also observed among those with atrial fibrillation, ischemic heart disease, and congestive heart failure with cortical CMIs independent of vascular risk factors.

Table 4 details the association of subclinical cardiac biomarkers with cortical CMIs after excluding persons with clinically manifest cardiac diseases. Higher levels of NT-proBNP and hs-cTnT were significantly associated with increasing cortical CMI counts on the MRI independent of vascular risk factors. However, with regard to hs-cTnT, this association was attenuated in those with 3 or more CMIs on the MRI.

\begin{tabular}{lccl}
\hline \multicolumn{4}{l}{ Table 1. Baseline Characteristics of the Included and Excluded Study Participants } \\
\hline Characteristic & $\begin{array}{l}\text { Included } \\
(\mathrm{n}=243)\end{array}$ & $\begin{array}{c}\text { Excluded } \\
(\mathrm{n}=219)^{\mathrm{b}}\end{array}$ & P Value \\
\hline Age, mean (SD), y & $72.8(9)$ & $72.1(7.1)$ & .36 \\
\hline Male & $116(42.9)$ & $94(47.7)$ & .30 \\
\hline Hypertension & $186(76.5)$ & $146(66.7)$ & .02 \\
\hline Hyperlipidemia & $181(74.5)$ & $162(74)$ & .90 \\
\hline Type 2 diabetes & $90(37)$ & $72(32.9)$ & .35 \\
\hline Atrial fibrillation & $21(8.6)$ & $13(5.9)$ & .27 \\
\hline Ischemic heart disease & $26(10.7)$ & $17(7.8)$ & .28 \\
\hline Congestive heart failure & $5(2.1)$ & $2(0.9)$ & .32 \\
\hline
\end{tabular}

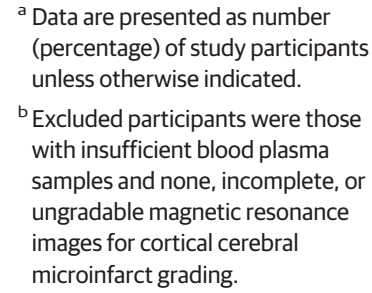
(percentage) of study participants unless otherwise indicated.

${ }^{\mathrm{b}}$ Excluded participants were those with insufficient blood plasma samples and none, incomplete, or ungradable magnetic resonance images for cortical cerebral microinfarct grading.

\begin{tabular}{|c|c|c|c|c|}
\hline Characteristic & $\begin{array}{l}\text { Participants } \\
\text { Without CMIs } \\
(n=173)\end{array}$ & $\begin{array}{l}\text { Participants } \\
\text { With } 1-2 \text { CMIs } \\
(n=46)\end{array}$ & $\begin{array}{l}\text { Participants } \\
\text { With } \geq 3 \text { CMIs } \\
(n=24)\end{array}$ & $\begin{array}{l}P \text { Value } \\
\text { for Trend }\end{array}$ \\
\hline \multicolumn{5}{|l|}{ Demographics } \\
\hline Age, mean (SD), y & $72.5(9.0)$ & $72.5(9.4)$ & $75.5(8.6)$ & .21 \\
\hline Male & $79(45.7)$ & $21(45.7)$ & $16(66.7)$ & .11 \\
\hline Hypertension & $127(73.4)$ & $37(80.4)$ & $22(91.7)$ & .04 \\
\hline Hyperlipidemia & $117(67.6)$ & $41(89.1)$ & $23(95.8)$ & $<.001$ \\
\hline Type 2 diabetes & $59(34.1)$ & $20(43.5)$ & $11(45.8)$ & .15 \\
\hline \multicolumn{5}{|l|}{ Cardiac factors } \\
\hline $\begin{array}{l}\text { NT-proBNP, median (IQR), } \\
\mathrm{pg} / \mathrm{mL}\end{array}$ & $113.3(152.7)$ & $135.8(268.9)$ & $791.3(1396.5)$ & .06 \\
\hline $\begin{array}{l}\text { High-sensitivity cardiac } \\
\text { troponin } \mathrm{T} \text {, median (IQR), } \\
\mathrm{ng} / \mathrm{mL}\end{array}$ & $0.010(0.008)$ & $0.011(0.011)$ & $0.016(0.012)$ & .007 \\
\hline Atrial fibrillation & $8(4.6)$ & $6(13)$ & $7(29.2)$ & $<.001$ \\
\hline Ischemic heart disease & $12(6.9)$ & $5(10.9)$ & $9(37.5)$ & $<.001$ \\
\hline Congestive heart failure & $1(0.6)$ & $1(2.2)$ & $3(12.5)$ & .001 \\
\hline \multicolumn{5}{|l|}{ MRI markers } \\
\hline $\begin{array}{l}\text { Total brain volume, mean } \\
\text { (SD), mL }\end{array}$ & $897.3(95.4)$ & $899.5(101.4)$ & $931.7(103.3)$ & .23 \\
\hline $\begin{array}{l}\text { White matter } \\
\text { hyperintensities, median } \\
\text { (IQR), mL }\end{array}$ & $4.37(11.50)$ & $4.58(11.1)$ & $11.8(33.4)$ & .06 \\
\hline Cerebral microbleeds & $84(50.6)$ & $26(63.4)$ & $17(73.9)$ & .02 \\
\hline Presence of lacunes & $50(29.4)$ & $16(35.6)$ & $15(65.2)$ & .002 \\
\hline $\begin{array}{l}\text { Presence of cortical } \\
\text { infarct }\end{array}$ & $18(10.6)$ & $6(13.3)$ & $15(65.2)$ & $<.001$ \\
\hline $\begin{array}{l}\text { Presence of intracranial } \\
\text { stenosis }\end{array}$ & $32(19.5)$ & $12(27.3)$ & $12(52.2)$ & .001 \\
\hline
\end{tabular}

Abbreviations: CMI, cortical cerebral microinfarct; IQR, interquartile range; $\mathrm{MRI}$, magnetic resonance imaging; NT-proBNP, N-terminal pro-brain natriuretic peptide.

SI conversion factors: To convert NT-proBNP to nanograms per liter, multiply by 1 ; high-sensitivity cardiac troponin T to micrograms per liter, multiply by 1 .

a Data are presented as number (percentage) of study participants unless otherwise indicated. 


\section{Discussion}

This study reveals that the circulating levels of cardiac biomarkers and clinically manifest cardiac diseases were associated with cortical CMI as detected on MRI in individuals attending a memory clinic. These associations were independent of other vascular risk factors. Furthermore, these cardiac biomarkers were also related to CMIs independent of clinically manifest cardiac diseases, suggesting that the subclinical cardiac dysfunction may contribute to the development of CMIs in the brain.

The secretion of NT-proBNP is primarily attributable to cardiomyocyte stretching, whereas hs-cTnT is a sensitive marker of myocardial injury. Previous studies have linked NTproBNP to atrial fibrillation, ${ }^{24}$ cognitive decline, and dementia. ${ }^{7,25,26}$ Earlier work ${ }^{10}$ has also found that NTproBNP and hs-cTnT were associated with cognitive impairment and dementia in patients with significant cerebrovascular diseases, independent of other vascular risk factors, suggesting that significant cerebral damage might have already taken place before the development of overt cardiac dis- eases. With respect to the clinically manifest cardiac diseases, several studies ${ }^{4,5}$ have found that coronary heart disease, atrial fibrillation, and congestive heart failure are linked to cognitive dysfunction through their role in causing cerebral damage. These cardiac diseases have also been suggested to be associated with other cerebrovascular diseases (strokes and white matter hyperintensities) through mechanisms such as cardioembolism and cerebral ischemic damage. Hence, early markers of cardiac dysfunction, such as NT-proBNP and hs-cTnT, are of interest so that timely intervention may be performed.

Previous studies ${ }^{13,15}$ of memory clinic patients and the general population have found that cortical CMIs were associated with large-vessel diseases (cortical infarcts and intracranial stenosis). Cortical CMIs in the right middle cerebral artery were more commonly seen in persons with right middle cerebral artery stenosis. The same was true with left middle cerebral artery stenosis. Furthermore, it was also reported that a history of cardiovascular diseases was associated with the presence of CMIs on MRI. ${ }^{15}$ Our present findings add to the previous literature by revealing a significant association of atrial fibrillation, ischemic heart disease, congestive heart failure, and subclinical cardiac biomarkers with CMIs independent of

\begin{tabular}{|c|c|c|c|}
\hline \multirow[b]{2}{*}{ Variable } & \multirow{2}{*}{$\begin{array}{l}\text { RR }(95 \% \mathrm{Cl}) \\
\text { for CMI Count }\end{array}$} & \multicolumn{2}{|l|}{ OR $(95 \% \mathrm{Cl})$} \\
\hline & & Presence vs Absence of CMls & $\geq 3$ vs $<3$ CMIs \\
\hline \multicolumn{4}{|c|}{ Subclinical Cardiac Diseases } \\
\hline \multicolumn{4}{|c|}{ Log pro-brain natriuretic peptide ${ }^{b}$} \\
\hline Model 1 & $3.85(3.18-4.66)$ & $2.71(1.49-4.93)$ & $5.34(2.37-12.06)$ \\
\hline Model 2 & $3.19(2.62-3.90)$ & $2.51(1.34-4.69)$ & $4.63(1.98-10.83)$ \\
\hline \multicolumn{4}{|c|}{$\begin{array}{l}\text { Log high-sensitivity cardiac } \\
\text { troponin } \mathrm{T}^{\mathrm{b}}\end{array}$} \\
\hline Model 1 & $7.98(5.08-12.53)$ & $3.99(1.30-12.22)$ & $13.23(2.38-73.64)$ \\
\hline Model 2 & $4.86(3.03-7.08)$ & $3.19(0.94-10.75)$ & $9.41(1.54-57.32)$ \\
\hline \multicolumn{4}{|c|}{ Clinical Cardiac Diseases } \\
\hline \multicolumn{4}{|c|}{ Atrial fibrillation } \\
\hline Model 1 & $2.14(1.59-2.88)$ & $4.42(1.73-11.32)$ & $4.99(1.72-14.47)$ \\
\hline Model 2 & $1.62(1.20-2.18)$ & $3.76(1.42-9.91)$ & $3.94(1.32-11.74)$ \\
\hline \multicolumn{4}{|c|}{ Ischemic heart disease } \\
\hline Model 1 & $5.61(4.41-7.14)$ & $3.28(1.43-7.54)$ & $7.24(2.67-19.63)$ \\
\hline Model 2 & $4.31(3.38-5.49)$ & $2.64(1.12-6.22)$ & $5.67(2.05-15.65)$ \\
\hline \multicolumn{4}{|c|}{ Congestive heart failure } \\
\hline Model 1 & $2.94(1.86-4.65)$ & $9.98(1.09-91.56)$ & $15.37(2.24-105.38)$ \\
\hline Model 2 & $2.05(1.29-3.25)$ & $7.23(0.78-66.77)$ & $10.68(1.54-74.06)$ \\
\hline
\end{tabular}

Table 4. Association of Subclinical Cardiac Biomarkers With CMls Excluding Participants With Clinically Manifest Cardiac Diseases ${ }^{a}$

\begin{tabular}{llll}
\hline & $\begin{array}{l}\text { RR }(95 \% \mathrm{CI}) \text { for CMI } \\
\text { Count }\end{array}$ & OR $(95 \% \mathrm{Cl})$ & \\
\cline { 4 - 4 } Variable & Presence vs Absence of CMIs & $\geq 3$ vs <3 CMIs \\
\hline $\begin{array}{l}\text { Log pro-brain natriuretic peptide } \\
\text { Model } 1\end{array}$ & $3.61(2.68-4.86)$ & $1.92(0.85-4.34)$ & $5.43(1.61-18.25)$ \\
\hline $\begin{array}{l}\text { Model } 2 \\
\text { Log high-sensitivity cardiac } \\
\text { troponin T }\end{array}$ & $3.16(2.33-4.27)$ & $1.93(0.83-4.48)$ & $4.80(1.37-16.82)$ \\
\hline $\begin{array}{l}\text { Model } 1 \\
\text { Model } 2\end{array}$ & $3.19(1.52-6.72)$ & $2.49(0.66-9.34)$ & \\
\hline
\end{tabular}

Abbreviations: CMI, cortical cerebral microinfarct; OR, odds ratio; RR, rate ratio.

${ }^{\text {a }}$ Model 1 was adjusted for age and sex. Model 2 was adjusted for age, sex, hypertension, and hyperlipidemia.

${ }^{\mathrm{b}}$ Interpretation of the effect sizes was as follows: a person with a 10 -fold (1 unit of 10-log [cardiac marker] increment) increase in cardiac biomarker level will be RR times (eg, 4 times) more likely to have 1 additional CMI on magnetic resonance imaging compared with a person with a lower biomarker level.

Abbreviations: $\mathrm{CMI}$, cortical cerebral microinfarct; $O R$, odds ratio; $R R$, rate ratio.

${ }^{\text {a }}$ Model 1 was adjusted for age and sex. Model 2 was adjusted for age, sex, hypertension, and hyperlipidemia. 
vascular risk factors. It has been postulated that the left ventricular dysfunction and ischemic heart disease trigger inflammatory changes that contribute to cerebral ischemic damage. ${ }^{27}$ Moreover, NT-proBNP has also been linked to subclinical brain injury (silent brain infarcts and white matter hyperintensities) ${ }^{8}$ and cardioembolic stroke. ${ }^{28}$ The stronger association of subclinical and clinically manifest cardiac diseases with 3 or more CMIs suggests that hypoperfusion and microemboli may be mechanisms for the development of CMIs. Indeed, the cause of CMIs is presumed to be heterogenous, with (micro) emboli, hypoperfusion, and microangiopathy as the leading cause of microinfarcts. ${ }^{29}$ The persistent association observed between cardiac biomarkers and CMIs in the absence of cardiac diseases further supports previous findings that circulating markers of cardiac dysfunction reflect silent brain injury or systemic vascular damage through shared vascular risk factors.

\section{Limitations}

Our study has several limitations. First, because these data were examined cross-sectionally, it is not possible to establish the temporal association between subclinical and clinical cardiac diseases with cortical CMIs on MRI. Second, because of the lower resolution of 3-T MRI, only approximately $25 \%$ of cortical CMIs seen on 7-T MRI are detectable on 3-T MRI. ${ }^{14}$ Hence, smaller cortical CMIs ( $<2 \mathrm{~mm}$ ) might have gone undetected, which may lead to underestimation of effect sizes. Third, because we did not have data on CMIs located at the border zone (because it is variable among individuals), we were unable to address whether CMIs in relation to cardiac disease specifically occur in vascular border zone areas. However, we have previously observed that CMIs are more likely to occur distally from intracranial stenosis, ${ }^{14}$ which further suggests that microemboli and hypoperfusion play an important role in their cause. Fourth, it remains to be investigated whether these findings are generalizable to other non-Asian populations with different vascular risk factor profiles. Strengths of the study include the wide availability of 3-T neuroimaging to grade cortical CMI, which makes it relatively feasible to study CMIs in large populations.

\section{Conclusions}

Higher levels of circulating markers of cardiac dysfunction and clinical cardiac diseases were associated with cortical CMI on MRI independent of vascular risk factors. Hence, early cardiac dysfunction may be a potentially modifiable treatment target to prevent CMI-related brain injury in patients attending a memory clinic. Further prospective studies are needed to understand the additional value of markers of cardiac dysfunction in the development of CMI and whether they may serve as surrogate outcomes in evaluating the effectiveness of novel treatment for cerebrovascular disease-related cognitive impairment.

\section{ARTICLE INFORMATION}

Accepted for Publication: November 1, 2016.

Published Online: February 6, 2017.

doi:10.1001/jamaneurol.2016.5335

Author Affiliations: Department of Pharmacology, Yong Loo Lin School of Medicine, National University of Singapore, Singapore (Hilal, Chai, Shaik, Chen); Memory Aging and Cognition Center, National University Health System, Singapore (Hilal, Chai, Shaik, Chen); Department of Radiology and Epidemiology, Erasmus Medical Center, Rotterdam, the Netherlands (Hilal); Department of Neurology, Brain Center Rudolf Magnus, University Medical Center Utrecht, Utrecht, the Netherlands (van Veluw, Biessels); Department of Neurology and Epidemiology, Erasmus Medical Center, Rotterdam, the Netherlands (Ikram); Raffles Neuroscience Centre, Raffles Hospital, Singapore (Venketasubramanian); Cardiovascular Research Institute, National University Heart Centre, Singapore (Richards).

Author Contributions: Dr Chen had full access to all the data in the study and takes responsibility for the integrity of the data and the accuracy of the data analysis.

Study concept and design: Hilal, Venketasubramanian, Biessels, Chen.

Acquisition, analysis, or interpretation of data: All authors.

Drafting of the manuscript: Hilal, Chen. Critical revision of the manuscript for important intellectual content: All authors.

Statistical analysis: Hilal.

Obtained funding: Chen.

Study supervision: Ikram, Venketasubramanian, Biessels, Chen.
Conflict of Interest Disclosures: None reported.

Funding/Support: This study was funded by grants NMRC/CG/NUHS/2010 and NMRC/CG/013/2013 from the Singapore National Medical Research Council (Dr Chen).

Role of the Funder/Sponsor: The funding source had no role in the design and conduct of the study; collection, management, analysis, and interpretation of the data; preparation, review, or approval of the manuscript; and the decision to submit the manuscript for publication.

\section{REFERENCES}

1. Cohen RA, Moser DJ, Clark MM, et al. Neurocognitive functioning and improvement in quality of life following participation in cardiac rehabilitation. Am J Cardiol. 1999;83(9):1374-1378.

2. Vogels RL, Oosterman JM, van Harten B, et al. Profile of cognitive impairment in chronic heart failure. J Am Geriatr Soc. 2007;55(11):1764-1770.

3. Hoth KF, Poppas A, Moser DJ, Paul RH, Cohen RA. Cardiac dysfunction and cognition in older adults with heart failure. Cogn Behav Neurol. 2008; 21(2):65-72.

4. Bell-McGinty S, Lopez OL, Meltzer CC, et al. Differential cortical atrophy in subgroups of mild cognitive impairment. Arch Neurol. 2005;62(9): 1393-1397.

5. Saykin AJ, Wishart HA, Rabin LA, et al. Olde adults with cognitive complaints show brain atrophy similar to that of amnestic $\mathrm{MCl}$. Neurology. 2006;67(5):834-842.

6. Gunstad J, Poppas A, Smeal S, et al. Relation of brain natriuretic peptide levels to cognitive dysfunction in adults $>55$ years of age with cardiovascular disease. Am J Cardiol. 2006;98(4) 538-540.

7. Kerola T, Nieminen T, Hartikainen S, Sulkava R, Vuolteenaho O, Kettunen R. B-type natriuretic peptide as a predictor of declining cognitive function and dementia: a cohort study of an elderly general population with a 5-year follow-up. Ann Med 2010;42(3):207-215.

8. Dadu RT, Fornage M, Virani SS, et al. Cardiovascular biomarkers and subclinical brain disease in the Atherosclerosis Risk in Communities Study. Stroke. 2013;44(7):1803-1808.

9. Webb IG, Yam ST, Cooke R, Aitken A, Larsen PD, Harding SA. Elevated baseline cardiac troponin levels in the elderly: another variable to consider? Heart Lung Circ. 2015;24(2):142-148.

10. Hilal S, Chai YL, Ikram MK, et al. Markers of cardiac dysfunction in cognitive impairment and dementia. Medicine (Baltimore). 2015;94(1):e297.

11. Prins ND, van Dijk EJ, den Heijer T, et al. Cerebral small-vessel disease and decline in information processing speed, executive function and memory. Brain. 2005;128(Pt 9):2034-2041.

12. Pantoni L, Poggesi A, Inzitari D. Cognitive decline and dementia related to cerebrovascular diseases: some evidence and concepts. Cerebrovasc Dis. 2009;27(suppl 1):191-196.

13. Hilal S, Sikking E, Chan QL, et al. Cortical cerebral microinfarcts on 3 Tesla magnetic resonance imaging: a novel marker for cognitive impairment [published online September 2, 2016]. Neurology. doi:10.1212/WNL.0000000000003110 
14. van Veluw SJ, Zwanenburg JJ, Engelen-Lee J, et al. In vivo detection of cerebral cortical microinfarcts with high-resolution 7T MRI. J Cereb Blood Flow Metab. 2013;33(3):322-329.

15. van Veluw SJ, Hilal S, Kuijf HJ, et al. Cortical microinfarcts on 3T MRI: clinical correlates in memory-clinic patients. Alzheimers Dement. 2015;11 (12):1500-1509.

16. Hilal S, Amin SM, Venketasubramanian $N$, et al. Subcortical atrophy in cognitive impairment and dementia. J Alzheimers Dis. 2015;48(3):813-823.

17. McKhann GM, Knopman DS, Chertkow H, et al. The diagnosis of dementia due to Alzheimer's disease: recommendations from the National Institute on Aging-Alzheimer's Association workgroups on diagnostic guidelines for Alzheimer's disease. Alzheimers Dement. 2011;7(3) 263-269.

18. Román GC, Tatemichi TK, Erkinjuntti T, et al. Vascular dementia: diagnostic criteria for research studies: report of the NINDS-AIREN International Workshop. Neurology. 1993;43(2):250-260.

19. World Medical Association. World Medical Association Declaration of Helsinki: ethical principles for medical research involving human subjects. JAMA. 2013;310(20):2191-2194. doi:10 .1001/jama.2013.281053
20. Ponikowski P, Voors AA, Anker SD, et al; Authors/Task Force Members; Document Reviewers. 2016 ESC Guidelines for the diagnosis and treatment of acute and chronic heart failure: the Task Force for the diagnosis and treatment of acute and chronic heart failure of the European Society of Cardiology (ESC): developed with the special contribution of the Heart Failure Association (HFA) of the ESC. Eur J Heart Fail. 2016;18(8):891-975.

21. Hilal S, Saini M, Tan CS, et al. Ankle-brachial index, cognitive impairment and cerebrovascular disease in a Chinese population. Neuroepidemiology. 2014:42(2):131-138

22. Wardlaw JM, Smith EE, Biessels GJ, et al; Standards for Reporting Vascular Changes on Neuroimaging (STRIVE v1). Neuroimaging standards for research into small vessel disease and its contribution to ageing and neurodegeneration. Lancet Neurol. 2013;12(8):822-838.

23. Cordonnier C, Potter GM, Jackson CA, et al. improving interrater agreement about brain microbleeds: development of the Brain Observer MicroBleed Scale (BOMBS). Stroke. 2009;40(1): 94-99.

24. Richards M, Di Somma S, Mueller C, et al. Atrial fibrillation impairs the diagnostic performance of cardiac natriuretic peptides in dyspneic patients: results from the BACH Study (Biomarkers in Acute Heart Failure). JACC Heart Fail. 2013;1(3):192-199.

25. Naito J, Naka Y, Watanabe H. Clinical impression of brain natriuretic peptide levels in demented patients without cardiovascular disease. Geriatr Gerontol Int. 2009;9(3):242-245.

26. Daniels LB, Laughlin GA, Kritz-Silverstein D, et al. Elevated natriuretic peptide levels and cognitive function in community-dwelling older adults. Am J Med. 2011;124(7):670.e1-670.e8.

27. Angermann CE, Frey A, Ertl G. Cognition matters in cardiovascular disease and heart failure. Eur Heart J. 2012;33(14):1721-1723.

28. Rost NS, Biffi A, Cloonan L, et al. Brain natriuretic peptide predicts functional outcome in ischemic stroke. Stroke. 2012;43(2):441-445.

29. Brundel M, de Bresser J, van Dillen JJ, Kappelle LJ, Biessels GJ. Cerebral microinfarcts: a systematic review of neuropathological studies. J Cereb Blood Flow Metab. 2012;32(3):425-436.

Copyright 2017 American Medical Association. All rights reserved. 